\title{
A STOCHASTIC VOLATILITY ALTERNATIVE TO SABR
}

\author{
L. C. G. ROGERS, ${ }^{*}$ University of Cambridge \\ L. A. M. VERAART, ${ }^{* *}$ Princeton University
}

\begin{abstract}
We present two new stochastic volatility models in which option prices for European plain-vanilla options have closed-form expressions. The models are motivated by the well-known SABR model, but use modified dynamics of the underlying asset. The asset process is modelled as a product of functions of two independent stochastic processes: a Cox-Ingersoll-Ross process and a geometric Brownian motion. An application of the models to options written on foreign currencies is studied.
\end{abstract}

Keywords: SABR; European option; volatility smile

2000 Mathematics Subject Classification: Primary 91B70

Secondary 60K99; 60J70; 62P05

\section{Introduction}

There is a growing interest in stochastic volatility models in all areas of financial mathematics; see, for example, [8], [12], [13], [14], [15], [17], [21], [23], and [25]. One stochastic volatility model which has gained great popularity with practitioners, in particular for modelling the foreign exchange market, is the so-called SABR model [11]. As presented in [11], it has the advantage that it allows asset prices and market smiles to move in the same direction. Moreover, a closed-form (approximate) formula for the implied volatility is given. This implied volatility is not constant but a function of the strike price and some other model parameters. Hence, the market prices and market risk, including vanna and volga risk, can be obtained very easily. Moreover, the SABR model is said to fit the implied volatility smile quite well. However, the SABR option pricing formula is not the option price corresponding to the underlying stochastic process, but is obtained by using an approximation, and as such must be treated with caution; the asymptotic is based on the assumption that the time-to-expiry is small, and the recent work of Benaim [3] showed that the extreme-strike behaviour of the formula is not consistent with arbitrage-free pricing.

The aim of this paper is to build an alternative model which retains many of the desirable features of the SABR model but also has exact closed-form expressions for the price of a European call option. The expressions involve a one-dimensional integral of elementary functions.

We begin by applying certain natural transformations to the SABR model, which suggest varying the model in such a way that the discounted asset price process becomes the product of two independent processes, whose transition densities are known in closed form. The explicit formulae for the option prices follow easily from this representation.

Received 15 April 2008; revision received 4 November 2008.

* Postal address: Statistical Laboratory, University of Cambridge, Wilberforce Road, Cambridge, CB3 OWB, UK.

Email address: 1.c.g.rogers@statslab.cam.ac.uk

** Current address: Institut für Stochastik, Universität Karlsruhe (TH), Kaiserstr. 89, 76133 Karlsruhe, Germany. 
We then generalise the model in Section 4. We define the discounted stock price as the product of a geometric Brownian motion and a function of the Cox-Ingersoll-Ross (CIR) process. The function used is essentially a confluent hypergeometric function. This choice makes the discounted asset price a martingale without restricting the choice of model parameters, creating a new model with seven parameters, in contrast to the four parameters of the SABR model, and the three of our original variant.

We therefore end up with a stochastic volatility model which is consistent with arbitrage-free pricing for all strikes and maturities. We do not rely on approximation techniques to derive the option prices for European plain-vanilla options, but get closed-form formulae. This is rarely possible for other stochastic volatility models. Another not too common feature of our model is the fact that we constructed an asset price process which is a martingale with finite higher moments and not just a local martingale; see, e.g. [2] and [22] for further discussion on this matter.

The recent preprint [16] presents a model similar to the ones we consider here.

\section{Motivation}

The SABR model is a stochastic volatility model in which the asset price and the volatility are correlated. The stock price $S$ is assumed to solve the stochastic differential equation (SDE)

$$
\mathrm{d} S=\sigma S^{\beta} \mathrm{d} W, \quad \mathrm{~d} \sigma=\eta \sigma \mathrm{d} B, \quad \mathrm{~d} B \mathrm{~d} W=\rho \mathrm{d} t,
$$

for some constants $\beta \in(0,1), \eta>0$, and $\rho \in(-1,1)$, where $W$ and $B$ are Brownian motions. In this model, singular perturbation techniques are used to obtain European option prices. Closed-form approximations to the option price and the implied volatility are stated in [11]. Here, we transform the basic SABR model, making various changes along the way, to arrive at a new model for which option prices are available in closed form. The prices are represented as one-dimensional integrals. It should be emphasised that this section is purely for motivation; we take the basic SABR model and carry out various transformations, changing the dynamics in various ways when it suits us, and making whatever simplifying choices appear helpful at the time. The reader for whom such free-form mathematics is anathema should immediately pass to the next section, where an explicit model is proposed ab initio, inspired by, but completely independent of, the account of this section.

Recall the constant elasticity of variance (CEV) model [5], where the stock price solves $\mathrm{d} S=\sigma S^{\beta} \mathrm{d} W$ for a constant $\sigma>0$. In this model it can be shown that the process $Y=$ $S^{\gamma}, \gamma=2(1-\beta)$, solves the SDE of a time-changed squared Bessel process. In particular, $Y_{t}=X\left(\gamma^{2} \sigma^{2} t / 4\right)$, where $X$ is a squared Bessel process with dimension $2\left(1-\gamma^{-1}\right)$, that is, $\mathrm{d} X=2\left(1-\gamma^{-1}\right) \mathrm{d} t+2 \sqrt{X} \mathrm{~d} \tilde{B}$; see, e.g. [7]. Then

$$
\mathrm{d} Y=\gamma \sigma \sqrt{Y} \mathrm{~d} W+\frac{1}{2} \gamma(\gamma-1) \sigma^{2} \mathrm{~d} t .
$$

Let us apply this transformation to the SABR model. We have to account for the fact that in the SABR model the two Brownian motions $B$ and $W$ are correlated. We therefore define the process $Y^{\prime}=y+\tilde{y}$ in terms of the processes

$$
\mathrm{d} y=\gamma \sigma \sqrt{y} \mathrm{~d} W^{\prime}+a \sigma^{2} \mathrm{~d} t, \quad \mathrm{~d} \tilde{y}=\gamma \sigma \sqrt{\tilde{y}} \mathrm{~d} B+b \sigma^{2} \mathrm{~d} t,
$$

where $W^{\prime}$ and $B$ are independent Brownian motions, and $a$ and $b$ are constants which sum to $\gamma(\gamma-1) / 2$. Then the correlation is $\mathrm{d} Y^{\prime} \mathrm{d} \sigma=\eta \sigma^{2} \gamma \sqrt{\tilde{y}} \mathrm{~d} t$ instead of $\mathrm{d} Y \mathrm{~d} \sigma=\eta \sigma^{2} \gamma \sqrt{Y} \rho \mathrm{d} t$, 
so that now the constant $\rho$ changes to the variable $\sqrt{\tilde{y} / Y}$. We can set some initial value for $\rho$ by our choice of $Y_{0}$ and $\tilde{y_{0}}$, but note that we cannot model negative correlation this way.

A particularly obliging choice of $b$ is to take $b=\gamma^{2} / 4$, since then

$$
\mathrm{d} \sqrt{\tilde{y}}=\frac{\sigma \gamma}{2} \mathrm{~d} B=\frac{\gamma}{2 \eta} \mathrm{d} \sigma
$$

one solution of which is

$$
\tilde{y}=\left(\frac{\gamma \sigma}{2 \eta}\right)^{2} .
$$

The corresponding choice for $a$ will be $a=-\beta(1-\beta)$, and if $x=y / \sigma^{2}$ then we find that

$$
\mathrm{d} x=\gamma \sqrt{x} \mathrm{~d} W^{\prime}-2 \eta x \mathrm{~d} B+\left(a+3 \eta^{2} x\right) \mathrm{d} t .
$$

For tractability, we propose instead to take $y=\sigma^{2} x^{\prime}$, where

$$
\mathrm{d} x^{\prime}=\gamma \sqrt{x^{\prime}} \mathrm{d} W^{\prime}+\left(a+3 \eta^{2} x^{\prime}\right) \mathrm{d} t,
$$

which is of course a different model, having the virtue that $x^{\prime}$ and $\sigma$ are independent. This leads to the model

$$
Y_{t}=y_{t}+\tilde{y}_{t}=\sigma_{t}^{2}\left(\left(\frac{\gamma}{2 \eta}\right)^{2}+x_{t}^{\prime}\right),
$$

where $\sigma$ and $x^{\prime}$ are independent. However, we will not necessarily have $Y^{1 / \gamma}$ to be a local martingale.

\section{First alternative to SABR}

\subsection{Model description}

Guided by the argument of the preceding section, we propose to represent the discounted asset price process by

$$
S_{t}=Y_{t}^{1 / \gamma}=\left(\sigma_{t}^{2} z_{t}\right)^{1 / \gamma}
$$

with $z$ and $\sigma$ the diffusions

$$
\mathrm{d} z=\left(a_{1}-a_{2} z\right) \mathrm{d} t+2 \sqrt{z} \mathrm{~d} W, \quad \mathrm{~d} \sigma=\eta \sigma \mathrm{d} B,
$$

where $0<\eta$ and $0<\gamma<2$ are constants, and $W$ and $B$ are two independent Brownian motions. The constants $a_{1}$ and $a_{2}$ are given by

$$
a_{1}=\frac{2(\gamma-1)}{\gamma}, \quad a_{2}=\frac{(2-\gamma) \eta^{2}}{\gamma},
$$

values which (as we will shortly see) make $S$ a martingale. The process $z$ is the squared modulus of a centred multidimensional Ornstein-Uhlenbeck (OU) process, commonly known in the finance literature as a CIR process.

Remark 1. If $a_{1}<0$, the process $z$ will hit 0 almost surely. Let $\tau:=\inf \left\{0 \leq t: z_{t}=0\right\}$. For $a_{1}<0$, we consider the stopped process $z_{t \wedge \tau}$ rather than $z$.

Definition 1. We refer to the model for the asset price defined by (1), (2), and (3) as the stochastic volatility model (SV1 model). 
We show in the following lemma that $S$ is a martingale.

Lemma 1. Suppose that the diffusions $z$ and $\sigma$ satisfy (2) and that the parameters are as in (3). Then the process $S_{t}=\sigma_{t}^{2 / \gamma} z_{t}^{1 / \gamma}$ is a martingale and solves the SDE

$$
\mathrm{d} S=S \frac{2}{\gamma}\left(\frac{\mathrm{d} W}{\sqrt{z}}+\eta \mathrm{d} B\right) .
$$

Proof. First we show that $S$ is a local martingale, using Itô calculus, and finally we argue a bound on $S$ to show that $S$ is a martingale.

Applying Itô's formula to the functions $x \mapsto x^{2 / \gamma}$ and $x \mapsto x^{1 / \gamma}$ we obtain

$$
\begin{aligned}
\mathrm{d} \sigma^{2 / \gamma} & =\sigma^{2 / \gamma}\left(\frac{2-\gamma}{\gamma^{2}} \eta^{2} \mathrm{~d} t+\frac{2 \eta}{\gamma} \mathrm{d} B\right) \\
\mathrm{d} z^{1 / \gamma} & =\frac{z^{1 / \gamma}}{\gamma}\left(\left(\frac{a_{1}}{z}-a_{2}+\frac{2(1-\gamma)}{\gamma z}\right) \mathrm{d} t+\frac{2}{\sqrt{z}} \mathrm{~d} W\right) .
\end{aligned}
$$

Using the product rule and the independence of the Brownian motions $B$ and $W$ gives

$$
\begin{aligned}
\mathrm{d} S & =\sigma^{2 / \gamma} \mathrm{d} z^{1 / \gamma}+z^{1 / \gamma} \mathrm{d} \sigma^{2 / \gamma} \\
& =S \frac{1}{\gamma}\left(\left(\frac{a_{1}}{z}-a_{2}+\frac{2(1-\gamma)}{\gamma z}+\frac{2-\gamma}{\gamma} \eta^{2}\right) \mathrm{d} t+\frac{2}{\sqrt{z}} \mathrm{~d} W+2 \eta \mathrm{d} B\right) .
\end{aligned}
$$

Substituting in the definitions of $a_{1}$ and $a_{2}$ we obtain

$$
\mathrm{d} S=S \frac{2}{\gamma}\left(\frac{1}{\sqrt{z}} \mathrm{~d} W+\eta \mathrm{d} B\right)=S \frac{2}{\gamma} \sqrt{\frac{1}{z}+\eta^{2}} \mathrm{~d} \tilde{W},
$$

where $\tilde{W}$ is a Brownian motion.

Then $S$ is a martingale, since $\sup _{0 \leq t \leq T} \sigma_{t} \in L^{p}$ and $\sup _{0 \leq t \leq T} z_{t} \in L^{p}$ for any $p>1$. Since $\sigma$ is a geometric Brownian motion, it is easy to see that $\sup _{0 \leq t \leq T} \sigma_{t} \in L^{p}$ for any $p>1$. Similarly, $\sup _{0 \leq t \leq T} z_{t} \in L^{p}$ for any $p>1$, since $\sup _{0 \leq t \leq T} z_{t}$ is bounded in law by the supremum of the squared Euclidean norm of an OU process in high enough dimension, as we show in the following.

Let $Y \in \mathbb{R}^{\delta}$ be a solution to the $\mathrm{SDE} \mathrm{d} Y_{t}=-\left(a_{2} / 2\right) Y_{t} \mathrm{~d} t+\mathrm{d} \hat{W}_{t}$, where $a_{2} \geq 0$ by construction and $\hat{W}$ is a $\delta$-dimensional Brownian motion. Let $f(\cdot): \mathbb{R}^{\delta} \rightarrow \mathbb{R}$ denote the squared Euclidean norm, i.e. $f(Y)=Y_{1}^{2}+\cdots+Y_{\delta}^{2}$. Then from Itô's formula we obtain $\mathrm{d} f(Y)=\left(\delta-a_{2} f\left(Y_{t}\right)\right) \mathrm{d} t+2 \sqrt{f\left(Y_{t}\right)} \mathrm{d} \tilde{W}_{t}$, where $\tilde{W}$ is a one-dimensional Brownian motion. Hence, we see that $z$ and $f(Y)$ have the same volatility function. Let $\delta_{1}:=\left\{g \in \mathbb{Z}: g \leq a_{1}<\right.$ $g+1\}+1$ and $\delta_{2}:=\left\{g \in \mathbb{Z}: g \leq a_{1}<g+1\right\}-1$. Therefore, $\delta_{1}-a_{2} z \geq a_{1}-a_{2} z \geq \delta_{2}-a_{2} z$ for all $z \geq 0$. Hence, by the stochastic comparison theorem (assuming that $f\left(Y_{0}\right)=z_{0}$ ) we constructed an almost sure upper bound and an almost sure lower bound on $z_{t}$ in terms of the squared Euclidean norm of an OU process in dimensions $\delta_{1}$ and $\delta_{2}$, respectively.

Before we can compute the prices of European put and call prices, we formulate the following lemma, which specifies the transition density of the process $z$.

Lemma 2. Suppose that the diffusion z satisfies (2). We define, for $t<T$,

$$
\begin{aligned}
c:=\frac{2 a_{2}}{4\left(1-\exp \left(-a_{2}(T-t)\right)\right)}, & u & :=c z_{t} \exp \left(-a_{2}(T-t)\right), \\
v & :=c z_{T}, & q:=\frac{a_{1}}{2}-1 .
\end{aligned}
$$


Then the following statements hold.

1. Given $z_{t}, z_{T}$ is distributed as $1 / 2 c$ times a noncentral $\chi^{2}$ random variable with $a_{1}$ degrees of freedom and noncentrality parameter $2 u$ :

$$
z_{T}=\frac{1}{2 c} \chi_{a_{1}}^{2}(2 u)
$$

2. For $a_{1}>0$, the transition density from $z_{t}$ to $z_{T}$ is given by

$$
p\left(z_{t}, z_{T}\right)=c \exp (-u-v)\left(\frac{v}{u}\right)^{q / 2} I_{q}(2 \sqrt{u v}),
$$

where $I_{q}(\cdot)$ denotes the modified Bessel function of the first kind of order $q$.

3. If $a_{1}<0$, the CIR process will hit 0 almost surely. Since we require that it is then absorbed at 0 , the distribution of $z$ has point mass $\Delta:=1-\int_{0}^{\infty} p\left(z_{t}, z\right) \mathrm{d} z>0$ at 0 . For $a_{1}<0$, the transition density is given by

$$
p\left(z_{t}, z_{T}\right)=c \exp (-u-v)\left(\frac{v}{u}\right)^{q / 2} I_{|q|}(2 \sqrt{u v}) .
$$

For additional information and a proof of the lemma, we refer the reader to [6], [9, Chapter 3.4], [10], and [18, Section 6.2.2].

In the following we exploit the independence of the two processes $\sigma$ and $z$, and compute prices for European put and call options by conditioning. This allows us to obtain analytic expressions for the option prices, as the next theorem states.

Theorem 1. (SV1 model.) Suppose that $S_{t}=\sigma_{t}^{2 / \gamma} z_{t}^{1 / \gamma}$, where the diffusions $z$ and $\sigma$ satisfy(2) and the parameters are as in (3). Let $r$ denote the interest rate, and let $\tilde{S}_{t}:=\mathrm{e}^{r t} S_{t}$ be the underlying asset price. Then the time-0-prices of a European put option, $P^{\mathrm{SV} 1}$, and of a European call option, $C^{\mathrm{SV} 1}$, with expiry $T$ and strike price $K$ are given

$$
\begin{aligned}
P^{\mathrm{SV} 1}\left(S_{0}, T, K, r, \eta, z_{0}, \gamma\right) & =\mathrm{E}\left[\left(\mathrm{e}^{-r T} K-S_{T}\right)^{+}\right] \\
& =\int_{0}^{\infty} h_{1}(z) p_{T}(z) \mathrm{d} z+\mathbf{1}_{\left\{a_{1}<0\right\}} \Delta \mathrm{e}^{-r T} K
\end{aligned}
$$

and

$$
C^{\mathrm{SV} 1}\left(S_{0}, T, K, r, \eta, z_{0}, \gamma\right)=\mathrm{E}\left[\left(S_{T}-\mathrm{e}^{-r T} K\right)^{+}\right]=\int_{0}^{\infty} h_{2}(z) p_{T}(z) \mathrm{d} z
$$

where

$$
\begin{aligned}
& h_{1}(z):=\mathrm{e}^{-r T} K \Phi\left(-d_{2}\right)-\sigma_{0}^{2 / \gamma} z^{1 / \gamma} \exp \left(\frac{\eta^{2} T}{\gamma}\left(\frac{2}{\gamma}-1\right)\right) \Phi\left(-d_{1}\right), \\
& h_{2}(z):=\sigma_{0}^{2 / \gamma} z^{1 / \gamma} \exp \left(\frac{\eta^{2} T}{\gamma}\left(\frac{2}{\gamma}-1\right)\right) \Phi\left(d_{1}\right)-\mathrm{e}^{-r T} K \Phi\left(d_{2}\right) .
\end{aligned}
$$

Here

$$
d_{1}:=d_{2}+\frac{2 \eta}{\gamma} \sqrt{T}, \quad d_{2}:=\frac{\gamma}{2 \eta \sqrt{T}}\left(\log \left(\frac{\sigma_{0}^{2 / \gamma} z^{1 / \gamma}}{\mathrm{e}^{-r T} K}\right)-\frac{\eta^{2}}{\gamma} T\right),
$$

$\Phi(\cdot)$ is the cumulative distribution function of the standard normal distribution, and $p_{T}(z):=$ $p\left(z_{0}, z_{T}\right)$ is the probability density function of the noncentral $\chi^{2}$ distribution, as specified in Lemma 2. 
Proof. We only show the expression for the put price, (4), since (5) is similar. We first assume that $a_{1} \geq 0$. From Lemma 1 we know that $S$ is a martingale. Hence, the put price is the expectation given in (4). Since the processes $z$ and $\sigma$ are independent, we can compute the expectation by conditioning as follows:

$$
\begin{aligned}
P^{\mathrm{SV} 1}\left(S_{0}, T, K, r, \eta, z_{0}, \gamma\right) & =\mathrm{E}\left[\left(\mathrm{e}^{-r T} K-S_{T}\right)^{+}\right] \\
& =\mathrm{E}\left[\left(\mathrm{e}^{-r T} K-\sigma_{T}^{2 / \gamma} z_{T}^{1 / \gamma}\right)^{+}\right] \\
& =\mathrm{E}\left[\mathrm{E}\left[\left(\mathrm{e}^{-r T} K-\sigma_{T}^{2 / \gamma} z_{T}^{1 / \gamma}\right)^{+} \mid z_{T}=z\right]\right] \\
& =\mathrm{E}\left[h_{1}(z)\right] \\
& =\int_{0}^{\infty} h_{1}(z) p_{T}(z) \mathrm{d} z
\end{aligned}
$$

where

$$
\begin{aligned}
h_{1}(z)= & \mathrm{E}\left[\left(\mathrm{e}^{-r T} K-\sigma_{T}^{2 / \gamma} z_{T}^{1 / \gamma}\right)^{+} \mid z_{T}=z\right] \\
= & \int_{-\infty}^{\infty}\left(\mathrm{e}^{-r T} K-\sigma_{0}^{2 / \gamma} z^{1 / \gamma} \exp \left(\left(\frac{2-\gamma}{\gamma^{2}} \eta^{2}-\frac{2 \eta^{2}}{\gamma^{2}}\right) T+\frac{2 \eta}{\gamma} \sqrt{T} x\right)\right)^{+} \\
& \quad \times \frac{\exp \left(-x^{2} / 2\right)}{\sqrt{2 \pi}} \mathrm{d} x \\
= & \int_{-\infty}^{a} \mathrm{e}^{-r T} K \frac{\exp \left(-x^{2} / 2\right)}{\sqrt{2 \pi}} \mathrm{d} x-\sigma_{0}^{2 / \gamma} z^{1 / \gamma} \exp \left(-\frac{\eta^{2} T}{\gamma}\right) \\
& \quad \times \int_{-\infty}^{a} \exp \left(-\frac{1}{2}\left(x^{2}-\frac{4 \eta \sqrt{T}}{\gamma} x\right)\right) \frac{\mathrm{d} x}{\sqrt{2 \pi}} \\
= & K \mathrm{e}^{-r T} \Phi(a)-\sigma_{0}^{2 / \gamma} z^{1 / \gamma} \exp \left(-\frac{\eta^{2} T}{\gamma}\right) \exp \left(\frac{2 \eta^{2} T}{\gamma^{2}}\right) \Phi\left(a-\frac{2 \eta \sqrt{T}}{\gamma}\right) \\
= & K \mathrm{e}^{-r T} \Phi(a)-\sigma_{0}^{2 / \gamma} z^{1 / \gamma} \exp \left(\frac{\eta^{2} T}{\gamma}\left(\frac{2}{\gamma}-1\right)\right) \Phi\left(a-\frac{2 \eta \sqrt{T}}{\gamma}\right)
\end{aligned}
$$

and

$$
a:=\frac{\gamma}{2 \eta \sqrt{T}}\left(\log \left(\frac{\mathrm{e}^{-r T} K}{\sigma_{0}^{2 / \gamma} z^{1 / \gamma}}\right)+\frac{\eta^{2} T}{\gamma}\right) .
$$

Then, with $d_{2}=-a$ and $d_{1}=-a+2 \eta \sqrt{T} / \gamma$, the result follows.

If $a_{1}<0$, the CIR process has point mass

$$
\Delta:=1-\int_{0}^{\infty} p\left(z_{t}, z\right) \mathrm{d} z>0
$$

at 0 and, therefore, the additional term $\Delta h_{1}(0)=\Delta \mathrm{e}^{-r T} K$ is added. Since, for the call price, $h_{2}(0)=0$, no additional term is necessary.

Integrals (4) and (5) can be rewritten as the definite integral

$$
\int_{0}^{1} h\left(\frac{1-x}{x}\right) p_{T}\left(\frac{1-x}{x}\right) \frac{\mathrm{d} x}{x^{2}},
$$

and can be evaluated by numerical integration. Alternatively, they can be evaluated by Monte Carlo methods by sampling from a noncentral $\chi^{2}$ distribution. 
Remark 2. In this model, the correlation between the asset and its volatility will always be positive, in contrast to the SABR model. Hagan et al. [11] stated that, with foreign exchange (FX) options, a key feature of the asset dynamics is that if the spot rises then the place where the implied volatility is minimal should also rise, and this was a feature that they claimed is not reflected by many stochastic volatility models. The model we propose here allows for this feature to some extent, since a shift of $\sigma$ has the effect of multiplying $S$ by some constant, but not altering the dynamics in any other way. Thus, if the spot moves upward due to an increase in $\sigma$ then the implied volatility surface also shifts to the right. However, the effect of a change in $z$ is ambivalent.

Remark 3. In the context of FX options, with $Y$ denoting the price of one unit of foreign currency in domestic currency units, $S_{t}=\exp \left(\left(r_{f}-r_{d}\right) t\right) Y_{t}$ is a martingale. Then the time0 -price of a European put option is given by

$$
\mathrm{E}\left[\exp \left(-r_{d} T\right)\left(K-Y_{T}\right)^{+}\right]=\exp \left(-r_{f} T\right) \mathrm{E}\left[\left(K \exp \left(-\left(r_{d}-r_{f}\right) T\right)-S_{T}\right)^{+}\right] .
$$

We denote the corresponding put and call prices in our model by $P^{\mathrm{SV} 1}\left(S_{0}, T, K, r_{d}, r_{f}, \eta, z_{0}, \gamma\right)$ and $C^{\mathrm{SV} 1}\left(S_{0}, T, K, r_{d}, r_{f}, \eta, z_{0}, \gamma\right)$, respectively.

\subsection{Empirical analysis}

In this section we present some empirical results. We consider data used in [4]. In the FX market, option prices are not quoted directly. The quotes are in terms of the Black-Scholes implied volatility. We consider EUR/USD volatility quotes as of 12 February 2004. On that day the spot exchange rate was 1.2832. The data contain observations for nine different maturities (one and two weeks; one, two, three, six, and nine months; and one and two years) and seven different strikes.

3.2.1. The fitting criterion. In the classical Black-Scholes model, the exchange rate process solves the SDE

$$
\mathrm{d} S^{\mathrm{BS}}=S^{\mathrm{BS}}\left(\left(r_{d}-r_{f}\right) \mathrm{d} t+\sigma^{\mathrm{BS}} \mathrm{d} W\right),
$$

where $r_{d}$ and $r_{f}$ denote the constant domestic and foreign interest rates, respectively. The volatility $\sigma^{\mathrm{BS}}$ is assumed to be constant. The price for a European call, $C^{\mathrm{BS}}$, and put, $P^{\mathrm{BS}}$, at time 0 with maturity $T$ and strike $K$ is then given by

$$
\begin{aligned}
& C^{\mathrm{BS}}\left(S_{0}, T, K, r_{d}, r_{f}, \sigma^{\mathrm{BS}}\right)=\exp \left(-r_{d} T\right)\left[S_{0} \exp \left(\left(r_{d}-r_{f}\right) T\right) \Phi\left(d_{1}^{\mathrm{BS}}\right)-K \Phi\left(d_{2}^{\mathrm{BS}}\right)\right], \\
& P^{\mathrm{BS}}\left(S_{0}, T, K, r_{d}, r_{f}, \sigma^{\mathrm{BS}}\right)=\exp \left(-r_{d} T\right)\left[K \Phi\left(-d_{2}^{\mathrm{BS}}\right)-S_{0} \exp \left(\left(r_{d}-r_{f}\right) T\right) \Phi\left(-d_{1}^{\mathrm{BS}}\right)\right],
\end{aligned}
$$

where

$$
d_{1,2}^{\mathrm{BS}}=\frac{\log \left(S_{0} / K\right)+\left(r_{d}-r_{f} \pm(1 / 2)\left(\sigma^{\mathrm{BS}}\right)^{2}\right) T}{\sigma^{\mathrm{BS}} \sqrt{T}},
$$

from which the implied volatilities are computed from the prices.

When looking at empirical data, we usually observe that the implied volatilities are not constant but U-shaped (volatility smile). In the FX market, this smile is usually symmetric. In the following we try to fit the SV1 model to the data such that we minimise the squared difference between the observed implied volatilities and the model implied volatilities. We denote by

$$
\sigma^{(\mathrm{SV} 1)}:=\sigma^{(\mathrm{SV} 1)}\left(\eta, z_{0}, \gamma\right)
$$


a model implied volatility, meaning that it solves

$$
P^{\mathrm{BS}}\left(S_{0}, T, K, r_{d}, r_{f}, \sigma^{(\mathrm{SV} 1)}\right)-P^{\mathrm{SV} 1}\left(S_{0}, T, K, r_{d}, r_{f}, \eta, z_{0}, \gamma\right)=0 .
$$

Suppose that $\left(\sigma_{1}^{\text {implied }}, \ldots, \sigma_{N}^{\text {implied }}\right)^{\top} \in \mathbb{R}^{N}$ is the vector containing the observed implied volatilities for European options corresponding to the vector $\left(K_{1}, \ldots, K_{N}\right)^{\top} \in \mathbb{R}^{N}$ of strike prices, the vector $\left(T_{1}, \ldots, T_{N}\right)^{\top} \in \mathbb{R}^{N}$ of maturities, and the vectors $\left(r_{d, 1}, \ldots, r_{d, N}\right)^{\top}$ and $\left(r_{f, 1}, \ldots, r_{f, N}\right)^{\top} \in \mathbb{R}^{N}$ of domestic and foreign interest rates, respectively. Here $S_{0}$ denotes the asset price at time 0 . In the following we minimise the squared difference between the observed implied volatility and the implied volatility derived from the model price, i.e. we compute

$$
\min _{\eta, z_{0}, \gamma} \sum_{i=1}^{N}\left(\sigma_{i}^{(\mathrm{SV} 1)}\left(\eta, z_{0}, \gamma\right)-\sigma_{i}^{\text {implied }}\right)^{2} .
$$

3.2.2. Implementation. The computation of the option price involves a numerical evaluation of the integral

$$
\int_{0}^{\infty} h(z) p_{T}(z) \mathrm{d} z
$$

see Theorem 1. This requires some care since in many examples the integrand has a very high and small peak. Simple integration routines might miss this point and might, therefore, compute too small prices. To overcome this problem, we did the following.

The integrand consists essentially of a product of a Black-Scholes-type formula $h(\cdot)$ and the density of a noncentral $\chi^{2}$ random variable $p_{T}(\cdot)$. So there are special functions involved: the cumulative distribution function of the standard normal distribution, $\Phi$, and the modified Bessel function of the first kind, $I_{q}$. Both functions can cause problems (regarding numerical precision) when considered with very small or large arguments. We therefore expressed $\Phi$ in terms of the logarithm of the complementary error function, $\log \left((2 / \sqrt{\pi}) \int_{x}^{\infty} \exp \left(-t^{2}\right) \mathrm{d} t\right)$. Moreover, we did not compute $I_{q}$ directly, but its scaled version $I_{q}(x) \mathrm{e}^{-|x|}$.

Then we considered the logarithm of the integrand rather than the integrand itself. We used an optimisation routine to determine the maximum of the logarithm of the integrand $x^{\star}$. We then split the area of integration and computed

$$
\int_{0}^{x^{\star}} h(z) p_{T}(z) \mathrm{d} z+\int_{x^{\star}}^{\infty} h(z) p_{T}(z) \mathrm{d} z .
$$

Therefore, we ensured that the numerical integration routine did not miss the main mass. The pricing routine was implemented in C using the GNU Scientific Library.

We used different optimisation routines to fit the data. We used gradient search methods, simulated annealing, and the simplex algorithm in [19].

3.2.3. Empirical results. In this section we present the empirical results from the analysis for one maturity (three months) only. The model parameters are as follows. The exchange rate at time 0 is $S_{0}=1.2832$, the maturity is $T=0.2493$, and the interest rates are $r_{d}=0.0112995$ and $r_{f}=0.0209007$. We consider seven observations. Figure 1 shows the results. We see that our model (SV1) and the SABR model seem to fit the European put prices well. However, if we consider the implied volatilities, we see that our model does not fit the implied volatilities as well as the SABR model does. The SABR model seems to fit the observed smile perfectly. However, we have to bear in mind that our model contains only three parameters, $\eta, z_{0}$, and $\gamma$ (since $\sigma_{0}=\sqrt{S_{0}^{\gamma} / z_{0}}$ ), whereas the SABR model contains four parameters. 


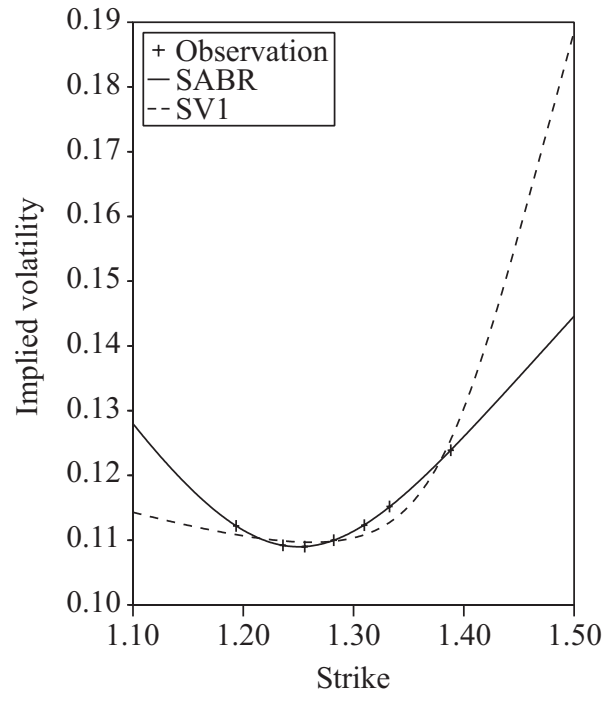

(a)

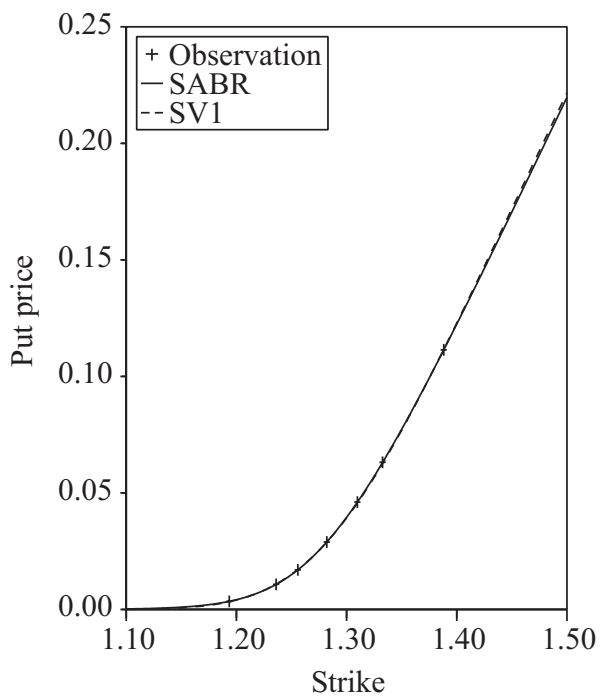

(b)

FIgURE 1: Comparing the fit of the SV1 model to the fit of the SABR model for (a) the implied volatilities and (b) the European put prices. The optimal parameters are $\beta=0.99, \eta=1.0052314, \sigma_{0}=0.1078418$, and $\rho=0.147685$ for the SABR model, and $\eta=0.01, z_{0}=91.576027$, and $\gamma=1.9980815$ for the

SV1 model.

\section{Second alternative to SABR}

\subsection{Model description}

We now generalise the approach of the previous section. We still assume that the discounted stock price can be written as a product of two independent processes. However, we now assume that the discounted stock price $S$ is a product of a geometric Brownian motion and a general function of a CIR process:

$$
S_{t}=\sigma_{t} g\left(z_{t}\right),
$$

where $\sigma$ is a geometric Brownian motion and $z$ is a CIR process, i.e.

$$
\mathrm{d} \sigma=\sigma(\mu \mathrm{d} t+\eta \mathrm{d} B), \quad \mathrm{d} z=\left(a_{1}-a_{2} z\right) \mathrm{d} t+2 \sqrt{z} \mathrm{~d} W .
$$

The two Brownian motions $B$ and $W$ are assumed to be independent, which makes the analysis tractable, but restricts the correlation between asset and volatility to be nonnegative. The function $g$ solves the following second-order ordinary differential equation (ODE):

$$
2 z g^{\prime \prime}(z)+\left(a_{1}-a_{2} z\right) g^{\prime}(z)+\mu g(z)=0 .
$$

(Observe that $g(z)=z^{1 / \gamma}$ is a solution if $a_{1}=2\left(1-\gamma^{-1}\right)$ and $a_{2}=\mu \gamma$. With $\mu=$ $(2-\gamma) \eta^{2} / \gamma^{2}$, we see that the SV1 model is a special case of the SV2 model; see Definition 2, below.) The ODE (8) can be solved in terms of the Kummer functions $M(\cdot, \cdot, \cdot)$ and $U(\cdot, \cdot, \cdot)$; see [1]. The Kummer functions are defined by

$$
M(a, b, z):=\sum_{n=0}^{\infty} \frac{(a)_{n} z^{n}}{(b)_{n} n !},
$$


where $(a)_{n}=a(a+1)(a+2) \cdots(a+n-1),(a)_{0}=1$, and

$$
U(a, b, z):=\frac{\pi}{\sin (\pi b)}\left(\frac{M(a, b, z)}{\Gamma(1+a-b) \Gamma(b)}-z^{1-b} \frac{M(1+a-b, 2-b, z)}{\Gamma(a) \Gamma(2-b)}\right) .
$$

Then a solution $g$ to the ODE (8) is given by

$$
g\left(z, a_{1}, a_{2}, \mu\right)=C_{1} M\left(-\frac{\mu}{a_{2}}, \frac{a_{1}}{2}, \frac{a_{2} z}{2}\right)+C_{2} U\left(-\frac{\mu}{a_{2}}, \frac{a_{1}}{2}, \frac{a_{2} z}{2}\right)
$$

for some constants $C_{1}$ and $C_{2}$.

Definition 2. We refer to the model for the asset price defined by (6), (7), and (9) as the stochastic volatility model (SV2 model). Moreover, we require that $\mu<0, a_{1}>2$, and $a_{2}>0$ in the following.

With this choice of parameters, the CIR process $z$ stays away from 0 . For $\mu<0$, the Kummer function $M$ stays positive. In the empirical analysis, the constants $C_{1}$ and $C_{2}$ were chosen such that the function $g(\cdot)$ stays positive.

With this choice of $g$ we have found a martingale.

Lemma 3. Suppose that the diffusions $z$ and $\sigma$ satisfy (7) and that $g$ is given by (9). Then the process $S_{t}=\sigma_{t} g\left(z_{t}\right)$ is a martingale and solves the $S D E$

$$
\mathrm{d} S_{t}=S_{t}\left(\frac{g^{\prime}\left(z_{t}\right)}{g\left(z_{t}\right)} 2 \sqrt{z_{t}} \mathrm{~d} W_{t}+\eta \mathrm{d} B_{t}\right) .
$$

Proof. We will have need of the result stated in Proposition 1, which can be seen as an application of Theorem 1.3.5 of [24], though we present a direct proof in Appendix A.

For diffusion (14), below, on $I=(0, \infty)$, it is well known (see, for example, [20, Chapter V]) that 0 is inaccessible if and only if $s(0+)=-\infty$ and that $+\infty$ is inaccessible if and only if $s(\infty)=\infty$, where $s$ is the scale function defined up to irrelevant affine transformations by

$$
s^{\prime}(x)=\exp \left(-\int^{x} \frac{2 b(z)}{\sigma(z)^{2}} \mathrm{~d} z\right)
$$

Routine calculations prove that 0 and $\infty$ are inaccessible for the diffusion $z$ satisfying (7) provided that $a_{1} \geq 2$ and $a_{2}>0$; for this diffusion,

$$
\sigma(x)=2 \sqrt{x}, \quad b(x)=a_{1}-a_{2} x, \quad s^{\prime}(x)=x^{-a_{1} / 2} \exp \left(\frac{a_{2}}{2} x\right) .
$$

It only remains to analyse the scale function of the drift transformed version of the diffusion, for which

$$
\sigma(x)=2 \sqrt{x}, \quad \tilde{b}(x)=a_{1}-a_{2} x+\frac{4 x g^{\prime}(x)}{g(x)}, \quad \tilde{s}^{\prime}(x)=\frac{s^{\prime}(x)}{(g(x))^{2}} .
$$

This will require the asymptotics of the Kummer functions at 0 and $\infty$.

We use the first-order approximation of the Kummer functions. According to [1, Chapter 13], for $z>0$ and $z \rightarrow \infty$,

$$
M(a, b, z)=\frac{\Gamma(b)}{\Gamma(a)} \mathrm{e}^{z} z^{a-b}\left(1+O\left(|z|^{-1}\right)\right), \quad U(a, b, z)=z^{-a}\left(1+O\left(|z|^{-1}\right)\right) .
$$


Hence, for large $z$, we can write

$$
\begin{aligned}
g\left(z, a_{1}, a_{2}, \mu\right) & =C_{1} M\left(-\frac{\mu}{a_{2}}, \frac{a_{1}}{2}, \frac{a_{2} z}{2}\right)+C_{2} U\left(-\frac{\mu}{a_{2}}, \frac{a_{1}}{2}, \frac{a_{2} z}{2}\right) \\
& =O\left(\exp \left(\frac{a_{2} z}{2}\right) z^{-\mu / a_{2}-a_{1} / 2}\right) .
\end{aligned}
$$

From (10), (11), and (12), we see that

$$
\int^{\infty} \tilde{s}^{\prime}(x) \mathrm{d} x=+\infty
$$

All that remains is to show that $\int_{0+} \tilde{s}^{\prime}(x) \mathrm{d} x=+\infty$, and for this we need the asymptotics near 0 of $g$.

For small $z$, the Kummer functions can be approximated; see [1, Chapter 13]. For $|z| \rightarrow 0$, $M(a, b, 0)=1$. For $U(\cdot, \cdot, \cdot)$, there are several approximations dependent on the value of the second parameter; see [1, Chapter 13, Equations 13.5.6-13.5.11]. Also, the order of the approximation varies. Since we require $a_{1}>2$, we obtain, for small $z$,

$$
U\left(-\frac{\mu}{a_{2}}, \frac{a_{1}}{2}, \frac{a_{2}}{2} z\right)=\frac{\Gamma\left(a_{1} / 2-1\right)}{\Gamma\left(-\mu / a_{2}\right)}\left(\frac{a_{2}}{2} z\right)^{1-a_{1} / 2}+ \begin{cases}O\left(|z|^{a_{1} / 2-2}\right), & a_{1}>4, \\ O\left(\log \left|\frac{a_{2}}{2} z\right|\right), & a_{1}=4, \\ O(1), & 2<a_{1}<4 .\end{cases}
$$

Whichever of these obtains, we immediately see that

$$
g(z)=O\left(z^{1-a_{1} / 2}\right) \quad \text { as } z \rightarrow 0 .
$$

Combining (10), (11), and (13), we see that

$$
\int_{0+} \tilde{s}^{\prime}(x) \mathrm{d} x=+\infty
$$

and the proof is complete by applying Proposition 1 (see Appendix A).

Then the prices of European put and call options can again be derived in closed form by conditioning.

Theorem 2. (SV2 model.) Suppose that $S_{t}=\sigma_{t} g\left(z_{t}\right)$, where the diffusions $z$ and $\sigma$ satisfy (7) and $g$ is given by (9). Let $r$ denote the interest rate, and let $\tilde{S}_{t}:=\mathrm{e}^{r t} S_{t}$ be the underlying asset price process. Then the time-0-prices of a European put option, $P^{\mathrm{SV} 2}$, and of a European call option, $C^{\mathrm{SV} 2}$, with expiry $T$ and strike price $K$ are given by

$$
P^{\mathrm{SV} 2}\left(S_{0}, T, K, r, a_{1}, a_{2}, z_{0}, \mu, \eta\right)=\mathrm{E}\left[\left(\mathrm{e}^{-r T} K-S_{T}\right)^{+}\right]=\int_{0}^{\infty} \tilde{h}_{1}(z) p_{T}(z) \mathrm{d} z
$$

and

$$
C^{\mathrm{SV} 2}\left(S_{0}, T, K, r, a_{1}, a_{2}, z_{0}, \mu, \eta\right)=\mathrm{E}\left[\left(S_{T}-\mathrm{e}^{-r T} K\right)^{+}\right]=\int_{0}^{\infty} \tilde{h}_{2}(z) p_{T}(z) \mathrm{d} z
$$


where

$$
\begin{aligned}
& \tilde{h}_{1}(z):=\mathrm{e}^{-r T} K \Phi\left(-\tilde{d}_{2}\right)-\sigma_{0} g(z) \mathrm{e}^{\mu T} \Phi\left(-\tilde{d}_{1}\right), \\
& \tilde{h}_{2}(z):=\sigma_{0} g(z) \mathrm{e}^{\mu T} \Phi\left(\tilde{d}_{1}\right)-\mathrm{e}^{-r T} K \Phi\left(\tilde{d}_{2}\right) .
\end{aligned}
$$

Here

$$
\tilde{d}_{1}=\frac{1}{\eta \sqrt{T}}\left(\log \left(\frac{\sigma_{0} g(z) \mathrm{e}^{\mu T}}{K}\right)+\left(r+\frac{\eta^{2}}{2}\right) T\right), \quad \tilde{d}_{2}=\tilde{d}_{1}-\eta \sqrt{T},
$$

$\Phi(\cdot)$ is the cumulative distribution function of the standard normal distribution, and $p_{T}(z):=$ $p\left(z_{0}, z_{T}\right)$ is the probability density function of the noncentral $\chi^{2}$ distribution, as specified in Lemma 2.

Proof. From Lemma 3 we know that $S$ is a martingale. The remaining part of the proof is along the lines of the proof of Theorem 1 and, therefore, omitted.

Remark 4. In this stochastic volatility model, the option price is effectively an average of Black-Scholes prices. Recall that in the classical Black-Scholes model, where the stock price $S$ is given by

$$
S_{t}=S_{0} \exp \left(\left(r-\frac{\eta^{2}}{2}\right) t+\eta W_{t}\right)
$$

the put price is given by

$$
P^{\mathrm{BS}}\left(S_{0}\right):=\mathrm{e}^{-r T} K \Phi\left(-d_{2}^{\mathrm{BS}}\right)-S_{0} \Phi\left(-d_{1}^{\mathrm{BS}}\right),
$$

where

$$
d_{1}^{\mathrm{BS}}:=\frac{1}{\eta \sqrt{T}}\left(\log \left(\frac{S_{0}}{K}\right)+\left(r+\frac{\eta^{2}}{2}\right) T\right), \quad d_{2}^{\mathrm{BS}}:=d_{1}^{\mathrm{BS}}-\eta \sqrt{T} .
$$

Therefore, if we substitute $S_{0}$ in this formula by the random variable $\sigma_{0} \mathrm{e}^{\mu T} g(z)$, where $z$ is a noncentral $\chi^{2}$ distributed random variable, we find that $\tilde{h}_{1}(z)=P^{\mathrm{BS}}\left(\sigma_{0} \mathrm{e}^{\mu T} g(z)\right)$. Moreover,

$$
P^{\mathrm{SV} 2}\left(S_{0}, T, K, r, a_{1}, a_{2}, z_{0}, \mu, \eta\right)=\mathrm{E}\left[P^{\mathrm{BS}}\left(\sigma_{0} \mathrm{e}^{\mu T} g(z)\right)\right] .
$$

Remark 5. The new stochastic volatility model, SV2, contains seven model parameters: $a_{1}$, $a_{2}, z_{0}, \mu, \eta, C_{1}$, and $C_{2}$. Again, $\sigma_{0}$ can be derived from $\sigma_{0}=S_{0} / g\left(z_{0}\right)$.

Remark 6. This modelling approach can be modified by replacing the CIR process with an OU process. The corresponding function $g(\cdot)$ can then still be expressed in terms of the Kummer functions. For this extension, it is possible to allow for correlation between the Brownian motion driving the geometric Brownian motion and the Brownian motion driving the OU process. European option prices can still be obtained in closed form.

\subsection{Empirical results}

We now fit the second stochastic volatility model, SV2, to the same example considered already in the previous section. We consider a European put option with three months expiry. Figure 2 shows the implied volatilities and the fitted option prices compared to the observations and the SABR model. We find that both the SV2 and the SABR models fit the put prices well. However, the SABR model still seems to fit the implied volatility smile better. 


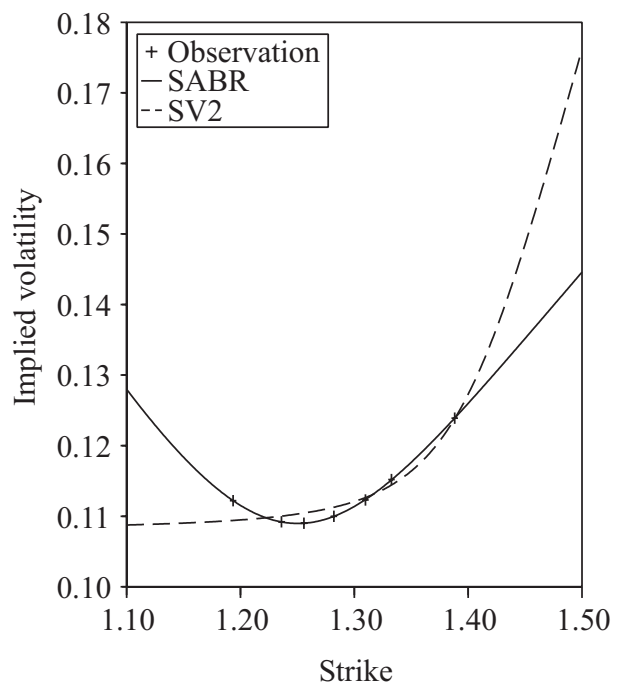

(a)

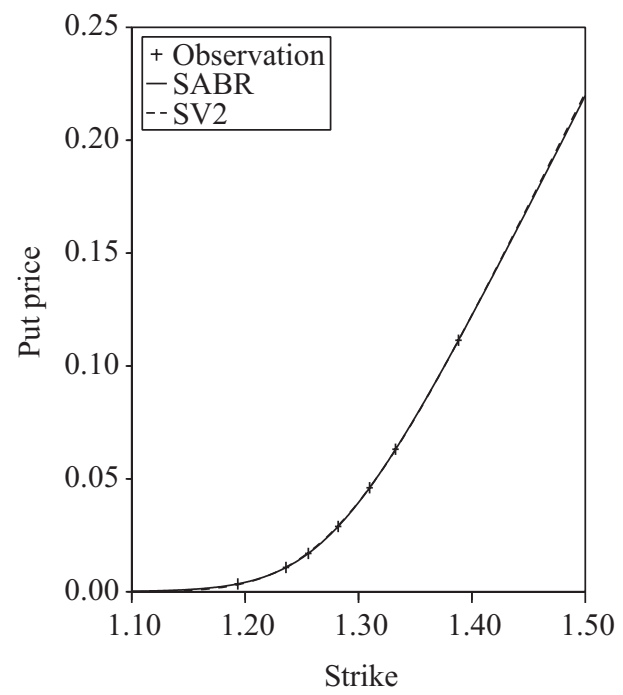

(b)

FIGURE 2: Comparing the fit of the SV2 model to the fit of the SABR model for (a) the implied volatilities and (b) the European put prices. The optimal parameters are $\beta=0.99, \eta=1.0052314$, $\sigma_{0}=0.1078418$, and $\rho=0.147685$ for the SABR model, and $a_{1}=3.977, a_{2}=0.849, \mu=$ $-0.0000439, \eta=0.1079, z_{0}=0.04305, C_{1}=1.57$, and $C_{2}=1.63$ for the SV2 model.

\section{Summary}

The aim of this paper was to construct a stochastic volatility which is close in spirit to the popular SABR model, but does not rely on approximation techniques. Moreover, we focused on the analytical and numerical tractability when choosing the dynamics and relationship of the stochastic processes involved. We obtained two stochastic volatility models which satisfy these criteria. In the first model, the discounted asset price is modelled as a product of two independent processes: a geometric Brownian motion and a power of a CIR process. In the second model, which generalises the first model, we expressed the discounted asset price as a product of two independent processes: a geometric Brownian motion and a confluent hypergeometric function of a CIR process. For both models, we derived analytic expressions for prices of European put and call options, which is rarely possibly in other stochastic volatility models. The prices can be expressed as integrals of elementary functions and can, therefore, be computed very efficiently. The models fitted well to FX option prices, and quite well to FX option implied volatilities.

\section{Appendix A. Result used in the proof of Lemma 3}

Proposition 1. Suppose that I is a nonempty, open interval and that $\sigma, b, \tilde{b}: I \rightarrow \mathbb{R}$ are locally Lipschitz in $I, \sigma>0$ throughout $I$. Let $\mathrm{P}$ and $\tilde{\mathrm{P}}$ be the laws on the path space $C\left(\mathbb{R}^{+}, I\right)$ under which the canonical process $X$ solves the SDEs

$$
\mathrm{d} X_{t}=\sigma\left(X_{t}\right) \mathrm{d} W_{t}+b\left(X_{t}\right) \mathrm{d} t, \quad X_{0}=x_{0},
$$

and

$$
\mathrm{d} X_{t}=\sigma\left(X_{t}\right) \mathrm{d} W_{t}+\tilde{b}\left(X_{t}\right) \mathrm{d} t, \quad X_{0}=x_{0},
$$


respectively, for some fixed $x_{0} \in I$. If $\tau:=\inf \left\{t: X_{t} \notin I\right\}$, and $Z$ is the 'change-of-measure' local martingale,

$$
\mathrm{d} Z_{t}=Z_{t} f\left(X_{t}\right) \mathrm{d} W_{t},
$$

where $f(x):=\sigma(x)^{-1}(\tilde{b}(x)-b(x))$, then $Z$ is a true martingale if and only if

$$
\tilde{\mathrm{P}}(\tau=\infty)=1 .
$$

Proof. First suppose that (15) holds. Take compact intervals $K_{n} \subset I$, increasing to $I$, and let $\tau_{n}=\inf \left\{t: X_{t} \notin K_{n}\right\}$. Then $Z_{t}^{n}:=Z_{t \wedge \tau_{n}}$ is a martingale for each $n$, because

$$
\mathrm{d} Z_{t}^{n}=Z_{t}^{n} f\left(X_{t}\right) \mathbf{1}_{\left\{t \leq \tau_{n}\right\}} \mathrm{d} W_{t}
$$

and the drift in the change of measure is bounded. Under the probability $\mathrm{P}^{n}$ given by

$$
\left.\frac{\mathrm{dP}^{n}}{\mathrm{dP}}\right|_{\mathcal{F}_{t}}=Z_{t}^{n},
$$

the process $X$ solves the SDE

$$
\mathrm{d} X_{t}=\sigma\left(X_{t}\right) \mathrm{d} W_{t}+\tilde{b}\left(X_{t}\right) \mathbf{1}_{\left\{t \leq \tau_{n}\right\}} \mathrm{d} t+b\left(X_{t}\right) \mathbf{1}_{\left\{t>\tau_{n}\right\}} \mathrm{d} t .
$$

Note that, for any $T \in \mathbb{R}^{+}$,

$$
\begin{aligned}
1 & =\mathrm{E}\left[Z_{T}^{n}\right] \\
& =\mathrm{E}\left[Z_{\tau_{n}}^{n}: \tau_{n} \leq T\right]+\mathrm{E}\left[Z_{T}^{n}: \tau_{n}>T\right] \\
& =\mathrm{E}\left[Z_{\tau_{n}}^{n}: \tau_{n} \leq T\right]+\tilde{\mathrm{P}}\left(\tau_{n}>T\right) \\
& =\mathrm{E}\left[Z_{\tau_{n}}: \tau_{n} \leq T\right]+\tilde{\mathrm{P}}\left(\tau_{n}>T\right) .
\end{aligned}
$$

Since $Z$ is a positive local martingale, it is a supermartingale, and so $1 \geq \mathrm{E}\left[Z_{T}\right]$. By hypothesis, $\tilde{\mathrm{P}}\left(\tau_{n}>T\right) \rightarrow 1$ as $n \rightarrow \infty$ and, therefore,

$$
\begin{aligned}
\mathrm{E}\left[Z_{T}\right] & =\mathrm{E}\left[Z_{T}: \tau_{n} \leq T\right]+\mathrm{E}\left[Z_{T}: \tau_{n}>T\right] \\
& =\mathrm{E}\left[Z_{T}: \tau_{n} \leq T\right]+\mathrm{E}\left[Z_{T}^{n}: \tau_{n}>T\right] \\
& =\mathrm{E}\left[Z_{T}: \tau_{n} \leq T\right]+\tilde{\mathrm{P}}\left(\tau_{n}>T\right) \\
& \rightarrow 1 .
\end{aligned}
$$

Conversely, if $Z$ is a martingale then the laws $\tilde{\mathrm{P}}$ and $\mathrm{P}$ are equivalent on each $\mathcal{F}_{t}$, so the event $\left\{\sup _{n} \tau_{n} \leq t\right\}$ has probability 0 under both $\mathrm{P}$ and $\tilde{\mathrm{P}}$.

\section{Acknowledgements}

The second author acknowledges support from the Engineering and Physical Sciences Research Council (UK) and the Deutsche Bank (London) under grant EPSRC CASE 05/004.

\section{References}

[1] Abramowitz, M. and Stegun, I. A. (1964). Handbook of Mathematical Functions with Formulas, Graphs, and Mathematical Tables (National Bureau Stand. Appl. Math. Ser. 55). US Government Printing Office, Washington.

[2] Andersen, L. And Piterbarg, V. (2007). Moment explosions in stochastic volatility models. Finance Stoch. 11, 29-50. 
[3] Benaim, S. (2007). Regular variation and smile asymptotics. Doctoral Thesis, University of Cambridge.

[4] Bisesti, L., Castagna, A. and Mercurio, F. (2005). Consistent pricing and hedging of an FX options book. Kyoto Econom. Rev. 74, 65-83.

[5] Cox, J. (1996). Notes on option pricing I: constant elasticity of variance diffusions. J. Portfolio Manag. 22, 15-17.

[6] Cox, J. C., Ingersoll, J. E., Jr. And Ross, S. A. (1985). A theory of the term structure of interest rates. Econometrica 53, 385-407.

[7] Delbaen, F. and Shirakawa, H. (2002). A note on option pricing for the constant elasticity of variance model. Asia-Pacific Financial Markets 9, 85-99.

[8] Dupire, B. (1992). Arbitrage pricing with stochastic volatility. In Proc. AFFI Conf. (June 1992), Paris.

[9] Glasserman, P. (2004). Monte Carlo Methods in Financial Engineering (Appl. Math. 53). Springer, New York.

[10] GöIng-Jaeschke, A. AND Yor, M. (2003). A survey and some generalizations of Bessel processes. Bernoulli 9, 313-349.

[11] Hagan, P. S., Kumar, D., Lesniewski, A. S. and Woodward, D. E. (2002). Managing smile risk. Wilmott Magazine, pp. 84-108.

[12] Heston, S. (1993). A closed-form solution for options with stochastic volatility with applications to bond and currency options. Rev. Financial Studies 6, 327-343.

[13] Hofmann, N., Platen, E. And Schweizer, M. (1992). Option pricing under incompleteness and stochastic volatility. Math. Finance 2, 153-187.

[14] Hull, J. And White, A. (1987). The pricing of options on assets with stochastic volatilities. J. Finance 42, 281-300.

[15] Hull, J. And White, A. (1988). An analysis of the bias in option pricing caused by a stochastic volatility. $A d v$. Futures Options Res. 3, 61.

[16] JäcKel, P. AND Kahl, C. (2007). Hyp hyp hooray. Preprint. Available at http://www.jaeckel.org/.

[17] Johnson, H. and Shanno, D. (1987). Option pricing when the variance is changing. J. Financial Quant. Anal. 22, 143-151.

[18] Lamberton, D. And Lapeyre, B. (1996). Introduction to Stochastic Calculus Applied to Finance. Chapman and Hall, London.

[19] Nelder, J. ANd Mead, R. (1965). A simplex method for function minimization. Comput. J. 7, $308-313$.

[20] Rogers, L. C. G. And Williams, D. (2000). Diffusions, Markov Processes and Martingales, Vol. 2. Cambridge University Press.

[21] Scotт, L. (1987). Option pricing when the variance changes randomly: theory, estimation, and an application. J. Financial Quant. Anal. 22, 419-438.

[22] Sin, C. A. (1998). Complications with stochastic volatility models. Adv. Appl. Prob. 30, 256-268.

[23] Stein, E. And SteIn, J. (1991). Stock price distributions with stochastic volatility: an analytic approach. Rev. Financial Studies 4, 727-752.

[24] Stroock, D. W. and Varadhan, S. R. S. (1979). Multidimensional Diffusion Processes. Springer, New York.

[25] Wiggins, J. (1987). Option values under stochastic volatility: theory and empirical estimates. J. Financial Econom. 19, 351-372. 